\title{
Hicksian Welfare Measures and the Normative Endowment Effect $t^{7}$
}

\author{
By Thomas A. Weber*
}

\begin{abstract}
We show that the Hicksian welfare measures of compensating variation and equivalent variation coincide if one of them is evaluated at a compensated income. The measures are nondecreasing in income if the varied attribute and income are complementary, and indirect utility is concave in income. Income monotonicity implies the normative endowment effect, where the equivalent variation exceeds the compensating variation. We provide sufficient conditions for the normative endowment effect and discuss empirical implications. In the global absence of a strict (anti-) endowment effect, both Hicksian welfare measures must be independent of income and the indirect utility function additively separable in income. (JEL D11, D63)
\end{abstract}

Tre

he equivalent variation and the compensating variation, introduced by John R. Hicks (1939), are used in demand theory to monetize the effect of a given welfare change on a consumer. The compensating variation $C$ corresponds to the consumer's willingness to pay (WTP) to effectuate the welfare change, while the equivalent variation $E$ measures the consumer's willingness to accept (WTA) to reverse the welfare change. In the interesting case where both measures are finite, the changes in the consumer's income prescribed by $C$ and $E$ exactly offset the welfare change either before or after it takes place. The monetary variations measure the income effect of the welfare change, either from an ex ante or an ex post perspective. More generally, both of these Hicksian welfare measures can be used for the evaluation of any change of state (which implies a change of welfare), as long as the agent's indirect utility for income is well-defined before and after the change.

Our aim is to examine the properties of WTA and WTP as a function of income and to clarify the normative relationship between them. The latter helps in refocussing empirical efforts to measure the well-known endowment effect. We provide an exact relation between compensating and equivalent variation (Proposition 2); one can be obtained from the other by evaluating it at a suitably compensated income. Knowing the corresponding "compensated-income function" is equivalent to knowing either one of the classical Hicksian welfare

\footnotetext{
* Department of Management Science and Engineering, Stanford University, Stanford, CA 94305-4121 (e-mail: webert@stanford.edu). I would like to thank Alexandre Kirillov and several anonymous referees for helpful comments. This research was supported in part by a Stanford Presidential Faculty Grant.

${ }^{\dagger}$ To comment on this article in the online discussion forum, or to view additional materials, visit the articles page at http://www.aeaweb.org/articles.php?doi=10.1257/mic.2.4.171.
} 
measures. For the comparison between $C$ and $E$, their monotonicity as a function of income is important. We show that while the compensated-income function is always increasing in income (Proposition 5), $C$ and $E$ are generally non-monotonic (Example 4). Nevertheless, monotonicity of one welfare measure implies monotonicity of the other (Corollary 6 and 7). As an interesting companion result to John K.-H. Quah (2007), we demonstrate that if the agent's indirect utility exhibits complementarities (between income and state change), and her indirect utility function is concave in income (at least in one state), then the welfare measures must be monotonic in income (Proposition 8).

Interestingly systematic differences (of arbitrary size) between the welfare measures, which we refer to as "normative (anti-)endowment effect" for $E$ greater (smaller) than $C$ at a given income level, are closely linked to monotonicity. The normative endowment effect is implied when the welfare measures are increasing in income (Proposition 14). The normative endowment effect also obtains when the compensated-income function satisfies a submodularity property (Proposition 16) and when income lies in the neighborhood of an "indifference point" (Proposition 18).

Extant empirical studies often classify disparities between the Hicksian welfare measures as behavioral anomalies. But WTA and WTP can be equal for all income levels only if they are both independent of income (Proposition 21), which is in turn equivalent to the additive separability of the consumer's indirect utility function in the state change and income. In that case, the sensitivity of her choice behavior to income must remain unaffected by the state change. Thus, observed differences between WTA and WTP can lead us to conclude that additive separability is not an appropriate assumption. Income compensation on a subject-specific transaction cycle allows us to test a behavioral anomaly with respect to the normative relationship between $C$ and $E$.

Related Literature.-The main difference between Hicks' welfare measures of compensating variation $C$ and equivalent variation $E$ is the reference point from which the variation is computed. Robert D. Willig (1976) noted that the difference between the two is likely to be small if the change in welfare is due to a price change of a market commodity. When the welfare change is due to a variation in the availability of a nonmarket good, W. Michael Hanemann (1991), based on earlier results by Alan Randall and John R. Stoll (1980), finds that the difference between $C$ and $E$ can be arbitrarily large (infinite in the limit), depending on the degree of substitutability between the nonmarket good and other ordinary market commodities. He restates the bounds on the difference obtained by Randall and Stoll (1980) in terms of elasticities, separating the influence of substitution and income effects. Weber (2003) establishes an exact relation between WTP and WTA (in integral form), given that the consumer's utility function is continuously differentiable. We argue that it does not really matter how a welfare change is effectuated, be it through a change in the prices of market goods, the provision of a nonmarket good, or through any other change in the boundary conditions. Without loss of generality, we take the nonmarket-goods perspective, and express the difference between $C$ and $E$ in terms of a compensated-wealth function. 
Many authors, starting with Judd Hammack and Gardner Mallard Brown, Jr. (1974); William D. Schulze, Ralph C. d'Arge, and David S. Brookshire (1981); and Jack L. Knetsch (1989), have empirically observed systematic differences between WTA and WTP when the welfare change is induced by the transfer of a nonmarket good. These experimental results, conducted in a variety of settings, suggest that a consumer's WTA tends to be larger than her WTP, a behavioral pattern that is generally referred to as the "endowment effect" or "status-quo bias." More recently, Charles R. Plott and Kathryn Zeiler $(2005,2007)$ note that the observed differences between WTA and WTP might disappear altogether if one controls for all possible biases in experimental settings, so that — as these authors point out - the endowment effect may be a mere artifact produced by observations under imperfect laboratory conditions.

The paper is organized as follows. In Section I, we provide an exact relation between the two welfare measures, and examine monotonicity as a key property. In Section II, we introduce the normative endowment effect and identify several conditions such as monotonicity of the welfare measures, submodularity of compensated income, or proximity to an indifference point that guarantee its presence. Section III discusses empirical implications, and Section IV concludes.

\section{Welfare Measures}

To position the results of this paper in the extant literature, we first present the standard model in the context of neoclassical demand theory. This motivating framework is inessential for the subsequent definitions and results.

\section{A. Motivation: The Classical Setup}

Suppose there are $n \geq 1$ conventional market goods, $x_{1}, \ldots, x_{n}$, and one nonmarket good $q .{ }^{1}$ We assume that the consumer's choice is determined by a rational, continuous, and locally nonsatiated preference relation $\preceq$ on the consumption set $\mathcal{X}=\mathbb{R}_{--}^{n+1}$, which is represented by the continuous, increasing utility function $u: \mathcal{X} \rightarrow \mathbb{R} \cdot{ }^{2}$ Given a vector $\mathbf{p}=\left(p_{1}, \ldots, p_{n}\right) \gg 0$ whose components represent prices for the respective market commodities, the consumer determines her Walrasian

\footnotetext{
${ }^{1}$ The restriction to a single nonmarket good is for expositional convenience only. Our analysis remains essentially unchanged if q denotes a vector of $m \geq 1$ nonmarket goods. The term "nonmarket good" is to be understood in a very general sense and could refer to any changes to the consumer's decision problem, such as price changes, changes to the choice set (e.g., due to limited product availability), or regulatory changes. For example, if one considers, instead of the provision of a nonmarket good, a price change from $\mathbf{p}$ to $\hat{\mathbf{p}}$, then $C(y)=e(\mathbf{p}, v(\mathbf{p}, y))-e(\hat{\mathbf{p}}, v(\mathbf{p}, y))$ and $E(y)=e(\mathbf{p}, v(\hat{\mathbf{p}}, y))-e(\hat{\mathbf{p}}, v(\hat{\mathbf{p}}, y))$, where the consumer's expenditure function at the price vector $\mathbf{p}$ and utility level $U$ is $e(\mathbf{p}, U)=\inf \left\{\mathbf{p} \cdot \mathbf{x}: u(\mathbf{x}) \geq U, \mathbf{x} \in \mathbb{R}_{+}^{n}\right\}$, and the indirect utility is $v(\mathbf{p}, y)=\sup \left\{u(\mathbf{x}): \mathbf{p} \cdot \mathbf{x} \leq y, \mathbf{x} \in \mathbb{R}_{+}^{n}\right\}$; cf. equations (1a) and (1b).

${ }^{2}$ A rational preference relation $\preceq$ is a binary relation defined for all elements of the choice space $\mathcal{X}$ that is reflexive (i.e., $\alpha \preceq \alpha$ holds for all $\alpha \in \mathcal{X}$ ), complete (i.e., $\alpha \preceq \beta$ or $\beta \preceq \alpha$ holds for any $\alpha, \beta \in \mathcal{X}$ ), and transitive (i.e., $\alpha, \beta, \gamma \in \mathcal{X}$, and $\alpha \preceq \beta \preceq \gamma$ implies that $\alpha \preceq \gamma$ ). The preference relation is continuous if for any $\alpha \in \mathcal{X}$ the upper contour set $U_{\alpha}=\{\beta \in \mathcal{X}: \alpha \preceq \beta\}$ and the lower contour set $L_{\alpha}=\{\beta \in \mathcal{X}: \beta \preceq \alpha\}$ are closed sets. It is locally nonsatiated if for any $\alpha \in \mathcal{X}$ and any $\varepsilon>0$ there exists a $\beta$ with $0<\|\alpha-\beta\|<\varepsilon$, such that $\alpha \preceq \beta$ and not $\beta \preceq \alpha$. A continuous preference relation can be represented by a continuous utility function $u: \mathcal{X} \rightarrow \mathbb{R}$, such that $\alpha \preceq \beta$ if and only if $u(\alpha) \leq u(\beta)$, for any $\alpha, \beta \in \mathcal{X}$. Local nonsatiation implies that the indirect utility (cf. footnote 1) is increasing in income.
} 
demand $\mathbf{x}^{*}(\mathbf{p}, q, y) \in \mathbb{R}_{+}^{n}$, subject to her finite income $y \geq 0$, by solving the classical utility maximization problem

$$
\max _{x \geq 0} u(q, \mathbf{x}), \text { subject to } \mathbf{p} \cdot \mathbf{x} \leq y,
$$

where $\mathbf{x}=\left(x_{1}, \ldots, x_{n}\right)$. Because of the continuity of $u$ and compactness of the choice set implied by the inequality constraints, there exists an optimal solution $\mathbf{x}^{*}(\mathbf{p}, q, y)$. Furthermore, as a consequence of local nonsatiation, which implies Walras' law (i.e., the income constraint is binding: $\mathbf{p} \cdot \mathbf{x}^{*}(\mathbf{p}, q, y)=y$ ), the consumer's indirect utility function,

$$
v(\mathbf{p}, q, y)=u\left(q, \mathbf{x}^{*}(\mathbf{p}, q, y)\right)
$$

is increasing in income, so that for any $(\mathbf{p}, q)$,

$$
y<\hat{y} \Rightarrow v(\mathbf{p}, q, y)<v(\mathbf{p}, q, \hat{y}) \text {. }
$$

It is in this setting that the Hicksian welfare measures are usually introduced. To abstract from this specific model, in what follows, we shall suppress the dependence on the price vector $\mathbf{p}$, which is either held constant or becomes part of the welfare change. As already noted in footnote 1, practically no assumption will be made about the nature of the state change under consideration.

\section{B. Definitions}

Consider a change in the state of an economy, characterized by the variation of an "instrument" $q$ from $q_{0}$ to $q_{1}$. As pointed out in Section IA, the change of state may correspond, for example, to a fluctuation in the availability of a nonmarket good, or it may be caused by a price change (cf. footnote 1 ). The precise nature of the state change does not matter, except with respect to the local-nonsatiation requirement that the agent's marginal value for income be positive before and after the state change. If $v_{i}(y)=v\left(q_{i}, y\right)$, for $i \in\{0,1\}$, denotes an agent's indirect utility for the state $q_{i}$, as an increasing function of income $y \in \mathbb{R},{ }^{3}$ we can define the Hicksian welfare measures relative to the change from $q_{0}$ to $q_{1}$ as follows: the compensating variation $C(y)$ is the maximum amount that the agent would want to pay for this change,

$$
C(y)=\sup \left\{c \in \mathbb{R}: v_{0}(y) \leq v_{1}(y-c)\right\}
$$

while the equivalent variation $E(y)$ is the minimum amount the agent would need to be paid to reverse the change after it has already taken place,

$$
E(y)=\inf \left\{e \in \mathbb{R}: v_{1}(y) \leq v_{0}(y+e)\right\}
$$

\footnotetext{
${ }^{3}$ In contrast to the classical setup in Section IA, income $y$ here is not assumed a priori bounded and can therefore take on any real value.
} 
Hence, any finite values of $E(y)$ and $C(y)$ can be determined using the implicit equations

$$
v_{0}(y)=v_{1}(y-C(y)),
$$

and

$$
v_{1}(y)=v_{0}(y+E(y))
$$

respectively. It follows directly from equations (1a) and (1b) that compensating and equivalent variations remain unaffected by an increasing transformation of the agent's utility function. In other words, the Hicksian welfare measures $C$ and $E$ are invariant with respect to the cardinal properties of the utility representation of the agent's preferences.

The compensated income $w\left(q_{0}, q_{1} ; y\right)$ for a change from $q_{0}$ to $q_{1}$ at income $y$ is such that it offsets the utility-impact of the change by varying income accordingly, i.e.,

$$
v_{0}(y)=v_{1}\left(w\left(q_{0}, q_{1} ; y\right)\right) \text {, }
$$

so that

$$
w\left(q_{0}, q_{1} ; y\right)=y-C(y) \quad\left[\equiv w_{01}(y)\right]
$$

Similarly, by reversing the role of $q_{0}$ and $q_{1}$, we see that the compensated income for the change from $q_{1}$ to $q_{0}$ is

$$
w\left(q_{1}, q_{0} ; y\right)=y+E(y) \quad\left[\equiv w_{10}(y)\right]
$$

REMARK 1: As Haneman (1991) points out, the two Hicksian welfare measures $C$ and $E$ can differ by an arbitrarily large amount. In fact, either one, as well as their difference, can become infinitely large, e.g., when $v_{0}\left(\right.$ resp. $\left.v_{1}\right)$ is bounded from above (resp. below) as illustrated in Figure 1.

Since the nonmarket good is (by virtue of the monotonicity of $u$ in $q$ ) desirable, the indirect utility function is increasing in $q$, and consequently,

$$
q_{1} \geq q_{0} \Leftrightarrow C(y), E(y)>0 .
$$

In other words, an attractive change in the provision of the nonmarket good implies nonnegative welfare measures and vice versa.

\section{Exact Relations}

The Hicksian welfare measures $C$ and $E$ as well as the compensated-income functions $w_{01}$ and $w_{10}$ are equivalent, in the sense that knowing one of them as a function of income implies the others. 


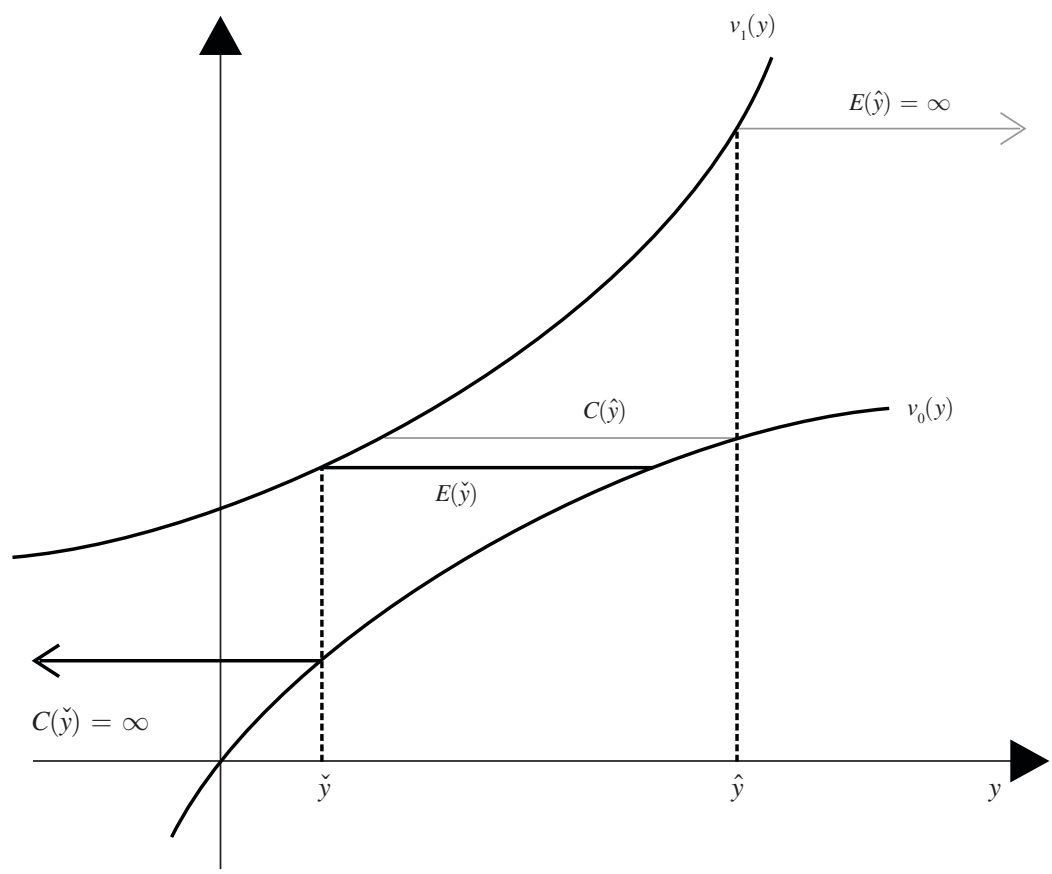

Figure 1. Possibility of Arbitrarily Large Differences between $C$ and $E$ ( $c f$. Remark 1$)$

PROPOSITION 2: Consider a state change from $q_{0}$ to $q_{1}$ at the income level $y$. Then

(i) $C(y)=E(y-C(y)) ; \quad\left[=E\left(w_{01}(y)\right)\right]$

(ii) $E(y)=C(y+E(y)) ; \quad\left[=C\left(w_{10}(y)\right)\right]$

(iii) $y=w_{10}\left(w_{01}(y)\right)=w_{01}\left(w_{10}(y)\right)$.

\section{PROOF:}

(i) From equations (1a) and (3a), we obtain that $v_{0}(y)=v_{1}(y-C(y))$ $=v_{1}\left(w_{01}(y)\right)$. By equations $(1 \mathrm{~b})$ and $(3 \mathrm{a})$, it is $v_{1}\left(w_{01}(y)\right)=v_{0}\left(w_{01}(y)+E\left(w_{01}(y)\right)\right)$ $=v_{0}(y-C(y)+E(y-C(y)))$. Since $v_{0}$ is increasing inincome, the equality of $v_{0}(y)$ and $v_{0}(y-C(y)+E(y-C(y)))$ implies that $y=y-C(y)+E(y-C(y))$.

(ii) As in the last part, applying equations (1b), (3b), (1a), (3b) in that order yields

$$
\begin{gathered}
v_{1}(y)=v_{0}(y+E(y))=v_{0}\left(w_{10}(y)\right)=v_{1}\left(w_{10}(y)-C\left(w_{10}(y)\right)\right) \\
=v_{1}(y+E(y)-C(y+E(y))),
\end{gathered}
$$

and, taking the inverse of $v_{1}$, therefore $y=y+E(y)-C(y+E(y))$. 
(iii) In the proof of part (ii), we had $v_{1}(y)=v_{1}\left(w_{10}(y)-C\left(w_{10}(y)\right)\right)$, so that, by taking the inverse of $v_{1}$ and using equation (3a), we get $y=w_{10}(y)-C\left(w_{10}(y)\right)$ $=w_{01}\left(w_{10}(y)\right)$. This means that the two compensated-income functions are inverses of each other, i.e., $w_{01}=w_{10}^{-1}$.

Proposition 2 establishes a direct relation between the Hicksian welfare measures $C$ and $E$, which is simpler than the one provided by Weber (2003), whose results appear in integral form and depend on additional smoothness assumptions on the agent's utility function.

REMARK 3: Applying Proposition 2, we can obtain from one of $C, E, w_{01}, w_{10}$ as a function of income all other functions. For example, if $C(\cdot)$ is given, then for any income level $y$, it is $w_{01}(y)=y-C(y), w_{10}(y)=w_{01}^{-1}(y) \in\{z \in \mathbb{R}: y=z-C(z)\}$, and $E(y)=C\left(w_{10}(y)\right)$. If $E(\cdot)$ is given, then $w_{10}(y)=y+E(y), w_{01}(y)=w_{10}^{-1}(y)$ $\in\{z \in \mathbb{R}: y=z+E(z)\}$, and $C(y)=E\left(w_{01}(y)\right)$. Finally, if a compensated-income function such as $w_{01}(\cdot)$ is known, then $w_{10}(y)=w_{01}^{-1}(y), C(y)=y-w_{01}(y)$, and $E(y)=w_{10}(y)-y$. Note that to determine the missing quantities we have actively used the fact that "global" information of one welfare measure is available, as a function of income.

The following example illustrates the concepts.

EXAMPLE 4: Consider an agent with the Cobb-Douglas utility function

$$
u(q, \mathbf{x})=k(q) x_{1}^{(1-\alpha)(1-q)} x_{2}^{\alpha(1-q)},
$$

where $\alpha \in(0,1)$ is a given constant, and $q \in[0,1]$ denotes the quantity of a certain nonmarket good. The function $k(q)$ is assumed to be increasing and positive-valued. Given any income level $y \in[0,1]$ and a fixed price vector $\mathbf{p}=\left(p_{1}, p_{2}\right) \gg 0$, the agent's Walrasian demand is

$$
\mathbf{x}^{*}(y)=\left(\frac{(1-\alpha) y}{p_{1}}, \frac{\alpha y}{p_{2}}\right) .
$$

Her indirect utility function becomes

$$
v(q, y)=u\left(q, \mathbf{x}^{*}(y)\right)=K(q) y^{1-q},
$$

which is nondecreasing in q, as long as

$$
K(q)=k(q)\left(\left(\frac{1-\alpha}{p_{1}}\right)^{1-\alpha}\left(\frac{\alpha}{p_{2}}\right)^{\alpha}\right)^{1-q}
$$

is nondecreasing in $q$. Let $0<q_{0}<q_{1}<1$. Then, 


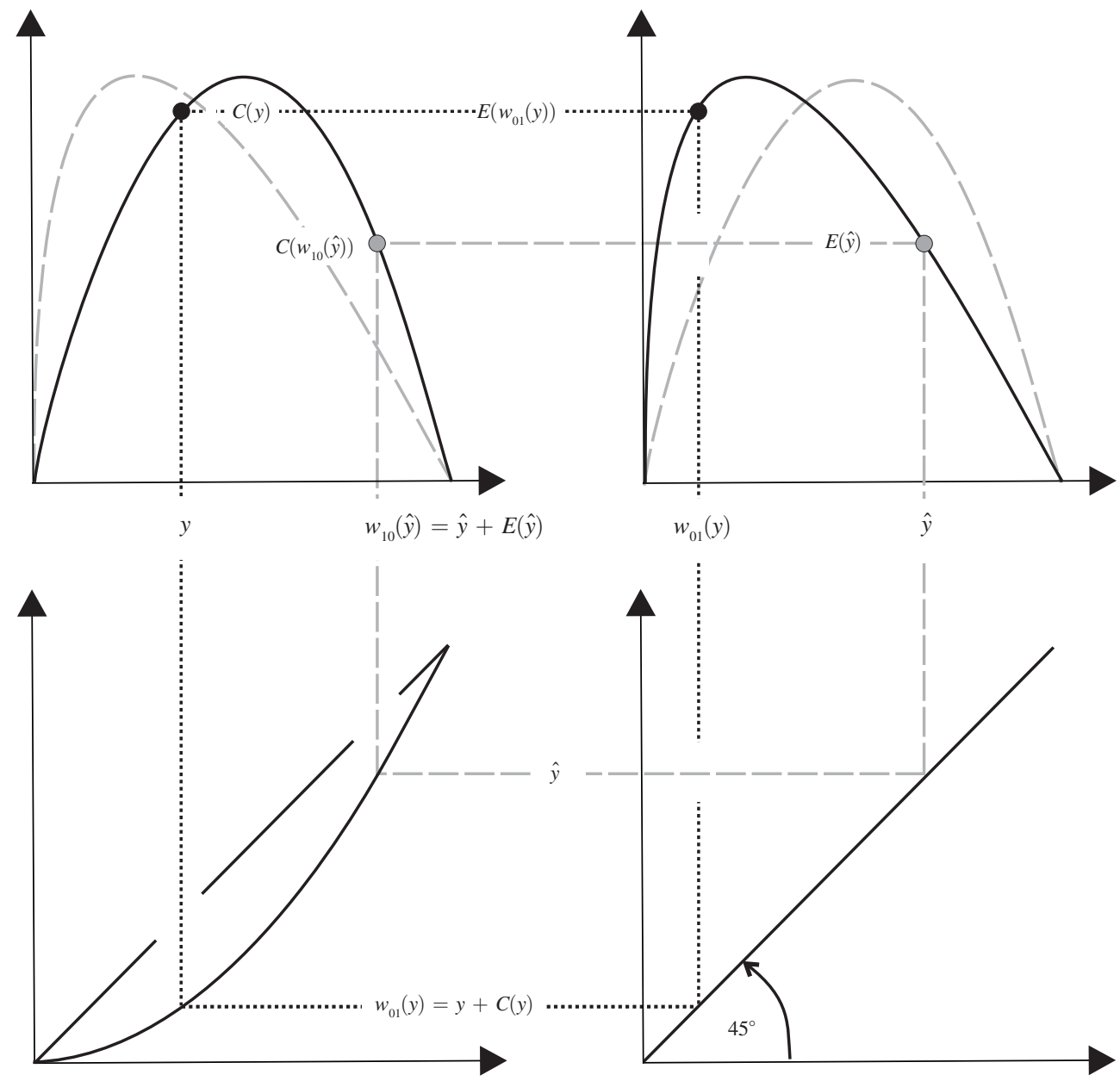

Figure 2. Compensating and Equivalent Variation in Example 4

$$
v\left(q_{1}, y-C(y)\right)=K\left(q_{1}\right)(y-C(y))^{1-q_{1}}=K\left(q_{0}\right) y^{1-q_{0}}=v\left(q_{0}, y\right),
$$

which yields the agent's compensating variation for a change from $q_{0}$ to $q_{1}$,

$$
C(y)=y-\left(\frac{K\left(q_{0}\right)}{K\left(q_{1}\right)}\right)^{\frac{1}{1-q_{1}}} y^{\frac{1-q_{0}}{1-q_{1}}}
$$

as a function of income. Using Remark 3, it is now straightforward to obtain expressions for the equivalent variation $E(y)$ as well as the compensated-income functions $w_{01}(y)$ and $w_{10}(y)$; $c f$. Figure 2.

\section{Monotonicity}

As can be observed in Example 4, the Hicksian welfare measures $C$ and $E$ are generally not monotonic in income. However, the degree of this non-monotonicity is limited by the strict monotonicity of the compensated-income functions. 
PROPOSITION 5: The compensated incomes $w_{01}(y)$ and $w_{10}(y)$ are increasing in $y$.

\section{PROOF:}

Fix arbitrary income levels $y, \hat{y}$ with $y<\hat{y}$. Since $v_{0}$ and $v_{1}$ are increasing, we obtain by equations $(1 \mathrm{a})$ and $(3 \mathrm{a})$ that $v_{1}\left(w_{10}(\hat{y})\right)=v_{0}(\hat{y})>v_{0}(y)=v_{1}\left(w_{10}(y)\right)$, and thus $w_{10}$ $(\hat{y})>w_{10}(y)$. Similarly, by equations $(1 \mathrm{~b})$ and $(3 \mathrm{~b})$ it is $v_{0}\left(w_{10}(\hat{y})\right)=v_{1}(\hat{y})>v_{1}(y)$ $=v_{0}\left(w_{10}(y)\right)$, so that $w_{10}(\hat{y})>w_{10}(y)$, concluding the proof.

Propositions 2 and 5 imply that the monotonicity of $C$ cannot obtain without the monotonicity of $E$ and vice versa.

COROLLARY 6: $C(y)$ is nondecreasing if and only if $E(y)$ is nondecreasing.

Locally, i.e., in the neighborhood of any given income level $y$, Corollary 6 does not have to hold, as can be seen in Figure 2, which depicts the situation of Example 4 in Section IC. It is also possible to sharpen the result somewhat by restricting attention to a subset of incomes.

COROLLARY 7: Let $\mathcal{Y} \subset \mathbb{R}$ be nonempty.

(i) $C$ is nondecreasing on $\mathcal{Y}$ if and only if $E$ is nondecreasing on $w_{01}(\mathcal{Y})$.

(ii) $E$ is nondecreasing on $\mathcal{Y}$ if and only if $C$ is nondecreasing on $w_{10}(\mathcal{Y})$.

The last result means that the welfare measure $C$ (resp. $E$ ) is (locally) nondecreasing at the income level $y$ if and only if $E$ (resp. $C$ ) is (locally) nondecreasing at the compensated income level $w_{01}(y)=y-C(y)\left(\operatorname{resp} . w_{10}(y)=y+E(y)\right)$.

We now turn our attention to the complementarity between income $y$ and the instrument $q$ as a sufficient condition for the Hicksian welfare measures $C$ and $E$ to be nondecreasing in income. In the sense of Francis Y. Edgeworth (1909) and Vilfredo F. D. Pareto (1897), we say that $q$ and $y$ are complementary if the indirect utility function $v(q, y)$ is supermodular in $(q, y)$, i.e., if

$$
\hat{q}>q, \hat{y}>y \Rightarrow v(\hat{q}, \hat{y})-v(q, \hat{y}) \geq v(\hat{q}, y)-v(q, y)
$$

If the indirect utility function $v$ is also concave in income for at least one of the two levels of the instrument $\left(q_{0}\right.$ or $\left.q_{1}\right)$, income monotonicity obtains.

PROPOSITION 8: Let the instrument and income be complementary. If, in addition, $v_{0}(y)$ or $v_{1}(y)$ is concave, then $E(y)$ and $C(y)$ are nondecreasing.

PROOF:

Fix any income levels $y, \hat{y}$ with $y<\hat{y}$, and assume that $v_{0}(\cdot)$ is concave. ${ }^{4}$ Then

$$
v_{0}(\hat{y})-v_{0}(y)=v_{1}(\hat{y}-C(\hat{y}))-v_{1}(y-C(y))
$$

\footnotetext{
${ }^{4}$ I am grateful to an anonymous reviewer for suggesting a proof much shorter than its original version.
} 


$$
\geq v_{0}(\hat{y}-C(\hat{y}))-v_{0}(y-C(y)) \text {, }
$$

where the equality is implied by the definition of $C$ in equation (1a), and the inequality follows from the complementarity of instrument and income, together with the fact that by Proposition 5 it is $w_{01}(\hat{y})=\hat{y}-C(\hat{y})>y-C(y)=w_{01}(y)$. By concavity of $v_{0}(\cdot)$, relation $(6)$ implies that

$$
\hat{y}-y \geq \hat{y}-C(\hat{y})-(y-C(y)),
$$

which simplifies to $C(\hat{y}) \geq C(y)$. If $v_{1}(\cdot)$ is concave, then we can show by an analogous argument that $E(\hat{y}) \geq E(y)$. From Corollary 6, we can therefore conclude that both $C(\cdot)$ and $E(\cdot)$ are nondecreasing.

EXAMPLE 9: In the context of Example 4, with $v(q, y)=y^{1-q}$ for $y \in[0,1]$ and $q \in\left\{q_{0}, q_{1}\right\}$, given $0<q_{0}<q_{1}<1$, we obtain from Proposition 8 that $($ since $v(q, \cdot)$ is concave) income monotonicity obtains when $v\left(q_{1}, y\right)-v\left(q_{0}, y\right)$ is nondecreasing in $y$. The latter holds if and only if $y \leq \bar{y}=\left(\left(1-q_{1}\right) /\left(1-q_{0}\right)\right)^{1 /\left(q_{1}-q_{0}\right)}$.

Since supermodularity is a cardinal rather than an ordinal property, it has been criticized by Hicks and R. G .D. Allen (1934) and Paul A. Samuelson (1947), among others, as not appropriate for characterizing complementarity in choice. Paul Milgrom and Chris Shannon (1994) provide an appropriate weakening of supermodularity in terms of quasi-supermodularity, which is an ordinal characteristic. ${ }^{5}$ For finite choice sets, Christopher P. Chambers and Federico Echenique (2009, theorem 1) show that a preference relation $\preceq$ over a finite choice set $\mathcal{X}$ has a quasi-supermodular and weakly increasing utility representation if and only if it also has a supermodular and weakly increasing utility representation, ${ }^{6}$ essentially elevating the combination of monotonicity and supermodularity to an ordinal property. In the setting with finite choice sets, the authors go on to show that supermodularity in conjunction with a weakly increasing utility representation is impossible to refute empirically. In other words, choice data generated from the maximization of any increasing utility function can also be generated using a supermodular increasing utility function. Their method is similar to S. N. Afriat's (1967) model, which showed that it is impossible to refute the hypothesis of a concave utility representation using any finite amount of data. However, while either concavity or supermodularity by itself might be a nonrefutable assumption, we have that, since together they imply normal demand (cf. Remark 10), they are empirically testable when combined. Hence, our assumption in Proposition 8 is refutable and therefore empirically testable. An easier way of seeing this is that it is clearly possible that $C(y)>E(y)$ (cf. Example 4), which by virtue of Proposition 14 implies that at least one of the two Hicksian welfare

\footnotetext{
${ }^{5}$ A function $u: \mathcal{X} \rightarrow \mathbb{R}$ is quasi-supermodular if for all $\alpha, \beta \in \mathcal{X}$ with $\alpha \wedge \beta, \alpha \vee \beta \in \mathcal{X}: u(\alpha) \geq(>)$ $u(\alpha \wedge \beta) \Rightarrow u(\alpha \vee \beta) \geq(>) u(\beta)$.

${ }^{6}$ The preference relation $\preceq$ on the choice set $\mathcal{X}$ has a weakly increasing utility representation if for all $\alpha, \beta \in \mathcal{X}: \alpha \preceq \beta \Rightarrow u(\alpha) \leq u(\beta)$. This is weaker than the ordinary definition of a utility representation (cf. footnote 2 ).
} 
measures is locally decreasing in income, thus refuting the combined assumption of concavity and supermodularity in Proposition 8.

REMARK 10: In the classical setting of Section IA, it is straightforward to verify that relation (5) holds if the agent's utility function $u(q, \mathbf{x})$ is supermodular and the demand $\mathbf{x}^{*}(q, y)$ is normal, that is, nondecreasing in $y$. Generalizing John $S$. Chipman's (1977) results, Quah (2007) shows that if $u(q, \mathbf{x})$ is supermodular and concave in $\mathbf{x}$, the agent's demand for market goods is normal.

The following two examples illustrate that monotonicity of Hicksian welfare measures can imply monotone comparative statics of economic aggregates with respect to shifts in the income distribution.

EXAMPLE 11: Let a change of the state q from 0 to 1 describe the possible provision of a public good. Consider a set of agents with incomes $y$ that are distributed with the cumulative distribution function $F(y)$ on a compact set $\mathcal{Y} \subset \mathbb{R}_{+}$. Each agent of income $y$ has willingness to pay $C(y)$ for the public good. The agents' aggregate value for the public good is therefore $W=\int_{\mathcal{y}} C(y) d F(y)$. Thus, if $C(\cdot)$ is increasing, then a first-order stochastically dominant (FOSD) shift of the income distribution, from $F$ to $\hat{F} \leq F$, increases the aggregate value for the public good from $W$ to $\hat{W} \geq W$.

EXAMPLE 12: Consider a market for a homogenous (private) good, in which agents have identical preferences, but different incomes $y$. Let $F(y)$ be the cumulative distribution function for the agents' income, and normalize the total number of agents to one. Suppose also that the willingness to pay $C(y)$ for the good, of which one can consume either zero $(q=0)$ or one $(q=1)$ unit, is increasing in an agent's income $y$. Then, at a price $p \in(0,1)$, the market demand, or, equivalently, the probability that an agent of random income $\tilde{y}$ purchases the good is $D(p)=\operatorname{Prob}(C(\tilde{y})$ $\geq p)=1-F\left(C^{-1}(p)\right)$. Hence, an FOSD shift in the income distribution increases the market demand for any price p, at least weakly. Note that a weaker (e.g., secondorder stochastically dominant) shift of the income distribution generically reduces the market demand at some price level. Similarly, if $C(y)$ is not monotonic, then at some price level the market demand may decrease despite an FOSD shift of the income distribution.

\section{Normative Endowment Effect}

We say that a normative endowment effect (NEE) is present at the income level $y$ if

$$
C(y) \leq E(y)
$$

The NEE is strict if the last inequality is strict. By equations (3a) and (3b), and the fact that $w\left(q_{0}, q_{0} ; y\right)=w\left(q_{1}, q_{1} ; y\right)=y$ inequality $(7)$ can be restated equivalently in terms of compensated-income functions, 
$\left(7^{\prime}\right)$

$$
C(y)=w\left(q_{1}, q_{1} ; y\right)-w\left(q_{0}, q_{1} ; y\right) \leq w\left(q_{1}, q_{0} ; y\right)-w\left(q_{0}, q_{0} ; y\right)=E(y) .
$$

Employing Proposition 2(iii) and the fact that, by virtue of equations (1a), (1b) and (3a), (3b), $w_{01}=v_{1}^{-1} \circ v_{0}$ and $w_{10}=v_{0}^{-1} \circ v_{1}$, it is useful to restate the last inequality in several equivalent ways,

$$
\begin{aligned}
2 y \leq w_{01}(y)+w_{10}(y) & =w_{01}(y)+w_{01}^{-1}(y)=w_{10}(y)+w_{10}^{-1}(y) \\
& =v_{0}^{-1}\left(v_{1}(y)\right)+v_{1}^{-1}\left(v_{0}(y)\right),
\end{aligned}
$$

emphasizing the dependence on income $y$ as well as the symmetry of the expressions on the right-hand side.

REMARK 13: After equations $(1 a),(1 b)$ we pointed out that the welfare measures are independent of the cardinal utility representation. Thus, without any loss in generality, we can choose the increasing transformation $v_{0}^{-1}$, and consider $\hat{v}_{0}(y)=v_{0}^{-1}\left(v_{0}(y)\right)$ $\equiv y$ and $\hat{v}_{1}(y) \equiv v_{0}^{-1}\left(v_{1}(y)\right) \equiv w_{10}(y)$. This transformation corresponds to choosing a money metric for the state $q_{0}$, where utility equals income, while at the final state utility equals the compensated income $w_{10}(y)$. Similarly, via the increasing utility transformation $v_{1}^{-1}$ one can use a money metric for the state $q_{1}$, such that $v_{0}(y)$ $\equiv w_{01}(y)$ and $v_{1}(y) \equiv y$. Under either money metric changes in the indirect utility, when switching between the states $q_{0}$ and $q_{1}$, correspond directly to the monetary WTA and WTP.

As the agent's income increases, it is natural to expect that her WTA and WTP for a "normal" (nonmarket) good $q$ also increase. It is therefore remarkable that the NEE follows from the monotonicity of the Hicksian welfare measures in income.

PROPOSITION 14: If $C(y)$ or $E(y)$ is nondecreasing, then $C(y) \leq E(y)$.

\section{PROOF:}

By Corollary 6, we have that $C(y)$ is nondecreasing if and only if $E(y)$ is nondecreasing. Thus, using Proposition 2 and the monotonicity of $C(y)$, it is 7

$$
E(y)-C(y)=C\left(w_{10}(y)\right)-C(y)=C(y+E(y))-C(y) \geq 0,
$$

which concludes our proof.

EXAMPLE 15: Consider a market for a homogeneous good, in which agents have identical preferences, but heterogeneous incomes (as in Example 12). Each agent is ex ante equally likely to be endowed with the good $(q=1)$ or not $(q=0)$. Once the endowments are realized, any agent can either do nothing or participate in the market to provide or procure a unit of the good. Normalizing the total mass

\footnotetext{
${ }^{7}$ One could use Proposition 2 to find that $E(y)-C(y)=E(y)-E\left(w_{01}(y)\right)=E(y)-E(y-C(y)) \geq 0$.
} 
of agents to two (i.e., mass one each for potential sellers and potential buyers), at a price $p$ the market demand is $D(p)=1-F\left(C^{-1}(p)\right)$, and the market supply is $S(p)=F\left(E^{-1}(p)\right)$, where we use the fact that by Corollary 6 the monotonicity of $C(y)$ implies the monotonicity of $E(y)$. For each endowment level $q \in\{0,1\}$, we can identify the set of transacting agents as a subset of $\mathcal{Y}=[0,1]$ : for $q=0$, active buyers in $\mathcal{Y}_{B}=\{y \in \mathcal{Y}: C(y) \geq p\}=\left[y_{B}, 1\right]$; for $q=1$, active sellers in $\mathcal{Y}_{S}=\{y: E(y) \leq p\}=\left[0, y_{S}\right]$. Since $y_{B}=C^{-1}(p)>y_{S}=E^{-1}(p)$, there exists a nonempty set of buyers $\mathcal{Y}_{N}=\left(y_{S}, y_{B}\right)$ who prefer not to trade at all. In equilibrium, demand equals supply, and the corresponding market-clearing condition, 1 $F\left(C^{-1}(p)\right)=F\left(E^{-1}(p)\right)$, implies a unique market price. In particular, if $C(y) \equiv E(y)$, then $p=0$ is the only market price at which trade can take place. Strict monotonicity of $C(\cdot)$ therefore implies gains from trade, as well as a nonempty "no-trade region" $\mathcal{Y}_{N}$ of intermediate incomes. Thus, in the presence of the normative endowment effect all active buyers have strictly higher income than all active sellers.

We now provide several additional conditions which guarantee the presence of the NEE.

Compensated-Income Criteria.-As becomes clear in Remark 13, compensated income is closely related to the primitives of the problem, with $w_{01}(y)=v_{1}^{-1}\left(v_{0}(y)\right)$ and $w_{10}(y)=v_{0}^{-1}\left(v_{1}(y)\right)$. Criteria for the NEE based on compensated income are therefore of immediate use.

PROPOSITION 16: Let $q_{1}>q_{0}$, and let $y$ be a given income level.

(I) The NEE obtains at income y if one of the following conditions holds:

$$
\varphi(2 y-\varphi(y)) \geq y \text { for some } \varphi \in\left\{w_{01}, w_{10}\right\}
$$

(ii) When $y \leq 0, w_{01}(y)=w_{10}(y)=y$; when $y>0$, there exists $\varepsilon \in[0,1]$ such that $w_{01}(y) \geq(1-\varepsilon) y$ and $w_{10}(y) \geq(1+\varepsilon) y$.

(iii) The compensated-income function $w(s, t ; y)$ is such that $\left(\partial^{2} w(s, t ; y) / \partial s \partial t\right) \leq 0$ for all $(s, t)$ in $\left[q_{0}, q_{1}\right] \times\left[q_{0}, q_{1}\right] . .^{8}$

(II) Condition (i) is necessary for the NEE to obtain at income $y$.

\section{PROOF:}

(i) By inequality (7') and the fact that $w_{10}=w_{01}^{-1}$ the NEE is present if and only if $\varphi(y)+\varphi^{-1}(y) \geq 2 y$. Condition (i) is a mere restatement of that inequality.

(ii) For $y \geq 0$, it is $w_{01}(y)+w_{10}(y) \geq(1-\varepsilon) y+(1+\varepsilon) y=2 y$, which yields the NEE via relation (7"). For $y<0$ the NEE obtains trivially.

\footnotetext{
${ }^{8}$ We implicitly assume here that $w(s, t ; y)$ is well-defined for any $s, t \in\left[q_{0}, q_{1}\right]$, allowing for convex combinations of states, and that it is twice continuously differentiable in $(s, t)$.
} 
(iii) The condition implies that the compensated-income function $w(s, t ; y)$ is submodular in $(s, t)$ on $\left[q_{0}, q_{1}\right] \times\left[q_{0}, q_{1}\right]$ (cf. Remark 17 below), which immediately implies relation (7').

REMARK 17: If for $q_{0}<q_{1}$, we set $\mathbf{q}=\left(q_{0}, q_{1}\right)$ and $\hat{q}=\left(q_{1}, q_{0}\right)$, then the NEE criterion (7') can be rewritten in the form

$$
w(\mathbf{q} \vee \hat{\mathbf{q}} ; y)+w(\mathbf{q} \wedge \hat{\mathbf{q}} ; y) \leq w(\mathbf{q} ; y)+w(\hat{\mathbf{q}} ; y)
$$

where $\vee$ and $\wedge$ are the "join" and "meet" operators used in lattice theory and the theory of monotone comparative statics (Topkis 1998) to denote the componentwise maximum and the componentwise minimum of two vectors, $\mathbf{q} \vee \hat{\mathbf{q}}=\max \{\mathbf{q}, \hat{\mathbf{q}}\}$, $\mathbf{q} \wedge \hat{\mathbf{q}}=\min \{\mathbf{q}, \hat{\mathbf{q}}\}$. The NEE is implied by submodularity of the compensated income $w(\cdot ; y)$. Note that condition (8) needs to hold only for the symmetric points $\left(q_{0}, q_{1}\right)$ and $\left(q_{1}, q_{0}\right)$, so that condition $\left(7^{\prime}\right)$ is in fact weaker than submodularity.

\section{B. Indifference Points}

Let us assume that the instrument $q$ is (strictly) desirable in the following sense: if $q_{1}>q_{0}$, then $v_{1}(y)(>) \geq v_{0}(y)$ for all income levels $y$. An income level $y_{0}$ is an indifference point with respect to the agent's preferences for the change in the provision of the instrument from $q_{0}$ to $q_{1}$ (or vice versa) if

$$
v_{0}\left(y_{0}\right)=v_{1}\left(y_{0}\right)
$$

The following result states that the difference between $C$ and $E$ is sign-definite in the right- and left-neighborhood of an indifference point.

PROPOSITION 18: Let $v_{0}, v_{1}$ be (real) analytic functions with the same image, i.e., such that $v_{0}(\mathbb{R})=v_{1}(\mathbb{R})$. If the instrument is desirable and $y_{0}$ is an indifference point, then there exist income levels $y_{-1}$ and $y_{1}$ with $y_{-1}<y_{0}<y_{1}$, such that the normative endowment effect is present for any income in $\left(y_{0,}, y_{1}\right)$ and the normative anti-endowment effect is present for any income in $\left(y_{-1}, y_{0}\right) .9$

\section{PROOF:}

See Appendix.

It is possible to view the last result as a corollary of our discussion of complementarity in Section ID. Because $v_{1} \geq v_{0}$, in the right-neighborhood of an indifference point $v_{1}-v_{0}$ must be nondecreasing, which, at least locally, implies a complementarity between income and the instrument. The presence of these complementarities in a right-neighborhood of the indifference point is independent of the utility representation of the agent's preferences. Hence, choosing a money metric with $v_{0}(y)=y$

\footnotetext{
${ }^{9}$ We say that a normative anti-endowment effect exists if for a given income level an agent's WTP exceeds her WTA.
} 


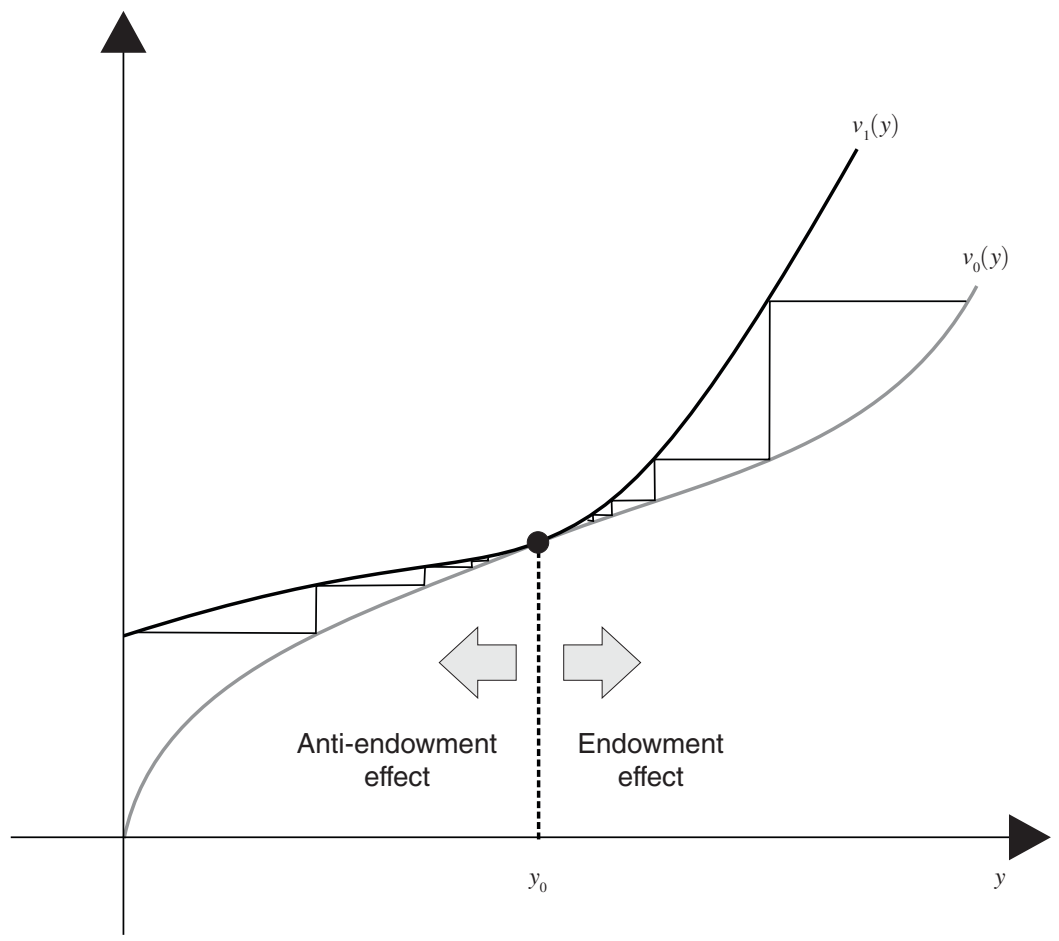

Figure 3. Endowment and Anti-Endowment Effect Near an Indifference Point $y_{0}$

ensures that the concavity assumption of Proposition 8 is automatically satisfied. Figure 3 depicts an indirect utility function that does not satisfy the assumptions of Proposition 14, but that in a right- and left-neighborhood of the indifference point generates a sign-definite difference between $C$ and $E .^{10}$

REMARK 19: Proposition 18 implies that if $y_{0}=0$ is an indifference point, then there exists a $y_{1}>0$, such that the NEE holds for all incomes in $\left(0, y_{1}\right)$.

Indifference points naturally arise in many decision problems, which is illustrated by the following example of the value of information in a classical portfolio investment problem.

EXAMPLE 20: An investor faces a decision about how much of her nonnegative income (wealth) y to save in a risk-free account at zero interest, and how much to invest in a risky asset of an uncertain return $\tilde{r}$ that is distributed with continuous distribution $F$ on the compact support $\left[r_{1}, r_{2}\right]$, given $r_{1}, r_{2}$ with $r_{1}<0<r_{2}$. Before

\footnotetext{
${ }^{10}$ The technical assumption that $v_{0}, v_{1}$ be analytic ensures that in any neighborhood of $y_{0}$ there can only be a finite number of roots of $v_{1}-v_{0}$, thus avoiding a familiar "shattering" phenomenon that could arise in a case where, e.g., $v_{1}(y)-v_{0}(y) \equiv y^{2} \sin ^{2}(1 / y)$, which is smooth, but not analytic. The latter assumption is without significant loss of generality, as the smoothness of an indirect utility function cannot be invalidated by means of any finite number of empirical observations. The assumption that $v_{0}$ and $v_{1}$ have the same image implies that the Hicksian welfare measures in equations (1a) and (1b) are finite.
} 
determining what amount $a \in[0, y]$ to invest in the risky asset (implying that $y-a$ is saved), the investor can observe the realization of a binary signal $\tilde{s}(q) \in\{L, H\}$, which is such that

$$
P(\tilde{s}(q)=L \mid \tilde{r} \leq 0)=P(\tilde{s}(q)=H \mid \tilde{r}>0)=q
$$

For $q=0.5$ the signal is completely uninformative, whereas for $q=1$, it corresponds to perfect information. The investor has a continuous increasing utility function $u(\mathbf{x})$ for monetary amounts $\mathbf{x} \in \mathbb{R}$. Maximizing the investor's expected utility yields the indirect utility $v(q, y)=V_{L}(q, y)+V_{H}(q, y)$, where, taking into account Bayes' rule and using the notation $p_{L}=1-q$ and $p_{H}=q$,

$$
V_{j}(q, y)=\max _{a \in[0, y]}\left\{\left(1-p_{j}\right) \int_{r_{1}}^{0} u(a r+y) d F(r)+p_{j} \int_{0}^{r_{2}} u(a r+y) d F(r)\right\}
$$

denotes the product of the investor's optimal utility and the probability $P(\tilde{s}(q)=j)$ of observing the signal realization $j \in\{L, H\}$. Let $q_{0}, q_{1} \in[0.5,1]$ with $q_{0}<q_{1}$. Then one can show that $\tilde{s}\left(q_{1}\right)$ is statistically sufficient for $\tilde{s}\left(q_{0}\right)$ (Richard E. Kihlstrom 1984), so that by David H. Blackwell's (1951) information order $v\left(q_{1}, y\right) \geq v\left(q_{0}, y\right)$, i.e., the investor prefers $\tilde{s}\left(q_{1}\right)$ to $\tilde{s}\left(q_{0}\right)$. The income level $y_{0}=0$ constitutes an indifference point, so that, by Proposition 18, the NEE must be present for all sufficiently small positive income levels, irrespective of any additional properties of the utility function or the return distribution F. For example, consider an investor with constant absolute risk aversion, so that $u(x)=1-\exp (-\rho x)$ for some $\rho>0$. Assume also that $0.5=q_{0}<q_{1}<1$ and $E[\tilde{r}]>0$. Then the investor seeks to invest the positive amount $a_{0}$ when observing any realization of the uninformative signal $\tilde{s}\left(q_{0}\right)$, and the amount $a_{j}$ when observing the realization $j \in\{L, H\}$ of the informative signal $\tilde{s}\left(q_{1}\right)$. Provided that the difference $q_{1}-q_{0}$ is small enough, we obtain that $0<a_{L} \leq a_{0} \leq a_{H}$. Thus, if $y \in\left[0, a_{L}\right]$, then also $a_{0}$ $=a_{H}=y$, implying that the investor is indifferent between $q_{0}$ and $q_{1}$. All incomes between 0 and $a_{L}$ are therefore indifference points, so that by Proposition 18 there exists $\varepsilon>0$, such that the NEE obtains for all $y \in\left[0, a_{L}+\varepsilon\right]$.

\section{Empirical Implications}

The first empirical disparities between WTP and WTA were noted in the valuation of public goods, such as hunting entitlements (Hammack and Brown 1974) or clean air (Schulze, d'Arge, and Brookshire 1981). In contexts with uncertainty Daniel Kahneman and Amos Tversky (1979) have observed that individuals tend to be risk-averse with respect to gains and risk-seeking with respect to losses, leading to a desire to avoid lotteries that tend to change the status quo, which Richard $\mathrm{H}$. Thaler (1980) terms the endowment effect. This has been confirmed experimentally by Knetsch and J. A. Sinden (1984). But uncertainty proves to be inessential for the observation of an endowment effect. In a famous experiment, Knetsch (1989) handed out coffee mugs to students and then offered them a large Swiss chocolate bar in exchange for the mug. He then repeated the experiment in a different class, 
starting with the chocolate bar, and subsequently offering coffee mugs in exchange. In both situations he found that only about 10 percent of the respondents were willing to trade. The experiments were repeated with money and two smaller candy bars, where different groups of individuals were asked to report their WTA and WTP for a one-way exchange, and significant differences between the two were observed. Knetsch notes that "as in the first test, the design of this experiment also precluded the (...) possibility of an income, or wealth, effect" (Knetsch 1989, 1279). One key point of our results is that this statement is not entirely correct. In the first experiment, one group is offered a trade from the state $\mathbf{q}^{0}=$ (coffee mug, no candy bar) to the state $\mathbf{q}^{1}=$ (no coffee mug, candy bar), while the second group is offered the opposite transfer, from $\mathbf{q}^{1}$ to $\mathbf{q}^{0}$. The second experiment is similar, only that now the coffee mug (or lack thereof) in both states of the world $\mathbf{q}^{i}$ is replaced by a fixed amount of money. Our discussion in Section II shows that, from a normative viewpoint, there is no reason to expect the transfers to be valued in the same way in both directions, and that it might therefore be possible that the observed behavior can still be described in terms of neoclassical choice theory. ${ }^{11}$

By labelling a positive difference between $E$ and $C$ endowment effect and calling it a behavioral anomaly (Kahneman, Knetsch, and Thaler 1991), virtually all extant empirical studies of differences between WTA and WTP implicitly assume that there are no income effects. Yet, observations at odds with an assumed lack of wealth effects cannot be taken as a significant piece of evidence for a behavioral anomaly, only as a failed test for the validity of this assumption. From a normative viewpoint, disconnecting WTA and WTP from any income effects arguably trivializes the agent's preferences. To see this, we first use the results in this paper to show that an identity between WTP and WTA implies that these Hicksian welfare measures have to be independent of income.

PROPOSITION 21: The following four statements are equivalent:

(i) $E(y)-C(y) \equiv$ const.

(ii) $C(y) \equiv E(y)$.

(iii) $C$ and $E$ are independent of income.

(iv) If $v_{i}(y) \equiv y$ for some $i \in\{0,1\}$, then $v_{1}(y)-v_{0}(y) \equiv$ const.

\section{PROOF:}

See Appendix.

\footnotetext{
${ }^{11}$ Our results apply a forteriori to Knetsch's third experiment which allowed for income effects, and indeed solicited them. In this test, subjects were asked if they would be willing to accept $\$ 700$ in exchange for a 0.5 percent increase of the chance of an accident that could require hospitalization, and others were asked if they would be willing to pay $\$ 700$ for a 0.5 percent decrease in the chance of such an accident. The author concludes from the observed differences in valuations that "[a]gain, relative preferences varied greatly depending on the direction of the proposed exchange" (Knetsch 1989, 1281-82), and finally that "reversibility will represent a normative ideal but will not serve as a very useful description of actual economic behavior" (Knetsch 1989, 1282). We argue here that Knetsch did not actually test for reversibility, since income compensation is required along the way.
} 
If the Hicksian welfare measures are independent of income, then the difference between the agent's indirect utility functions before and after the welfare change is also independent of income. This implies that the income dependence of the agent's other consumption decisions is unaffected by the welfare change. ${ }^{12}$ The converse also holds.

To be clear, we do not claim that previous experimental observations of the behavioral endowment effect are incorrect. Our results highlight the need to carefully control for the absence of income effects before it is possible to identify a behavioral anomaly that contradicts rational choice behavior. One way to do so is to examine both transitions with full income compensation, i.e., from $q_{0}$ to $q_{1}$ plus a money transfer and then back from $q_{1}$ to $q_{0}$ minus a money transfer, for the same individual. The money transfers must then normatively be identical, independent of the direction in which the transaction cycle is traversed. The difference between the extant literature on the endowment effect and the normative identity between the Hicksian welfare measures $C$ and $E$ is that income compensation of one welfare measure is required for the identity to hold, a fact that is neglected by all studies thus far.

In Section IC, we have shown that normatively an identity between $C$ and $E$ holds if one of the two Hicksian welfare measures is evaluated at an appropriately compensated income. Hence, instead of trying to control for income effects, orequivalently - assuming that the agent's preferences are actually separable between any market and nonmarket goods, one can simply move the frame of reference and test the normative identity.

Let us therefore consider the following revised version of the coffee mug experiment that attempts to test a behavioral anomaly relative to the relation $C(y)$ $\equiv E\left(w_{01}(y)\right)$, instead of the relation $C(y) \equiv E(y)$. For this, the experiment would elicit a subject's WTP for a nonmarket good (e.g., a special mug), making sure that there is no informational asymmetry about the good before the purchase and no other subject misconceptions. ${ }^{13}$ It should also be clear to the individual that the good can be sold in a second nonmarket transaction. Then, one can elicit an individual's WTP (at her income $y$ ) for this welfare change, using either direct or marketbased elicitation methods. The transaction should then be implemented, meaning that the individual pays WTP to the experimenter and obtains the nonmarket good. Subsequently, the experimenter elicits this subject's willingness to accept, at her compensated income, $w_{01}(y)=y-$ WTP for undoing the welfare change, which we denote by WTA'. Since by Proposition 2, at any income level $y$, we have that

$$
y=w_{10}\left(w_{01}(y)\right)=w_{01}(y)+E\left(w_{01}(y)\right)=y-C(y)+E\left(w_{01}(y)\right),
$$

\footnotetext{
${ }^{12}$ In the classical setting of Section IA, Proposition 21 below implies that if an agent's preference relation $\preceq$ is represented by a quasilinear utility function of the form $u(q, \mathbf{x})=a(q)+b(\mathbf{x})$, then $C(y) \equiv E(y)$.

${ }^{13}$ Plott and Zeiler (2005) examine the experimental procedures used in previous studies for eliciting the WTPWTA differences, and find that when they control for all possible subject misconceptions and at the same time use the market-based elicitation mechanism by Gordon M. Becker, Morris H. DeGroot, and Jacob Marschak (1964) to determine monetary values, then the differences between WTA and WTP disappear. Plott and Zeiler (2007) go on to examine various theoretical explanations for the endowment effect, which in conjunction with subject misconceptions introduced in the various experimental procedures, might have led previous authors to incorrectly interpret observations as manifestations of an endowment effect. None of their explanations overlap with our discussion of the normative differences between WTA and WTP.
} 
WTP and WTA' have to be equal from a normative standpoint. It is important to note that in this revised coffee mug experiment it is not necessary to elicit anything from the subject other than her WTP and her WTA' at the compensated income after her paying WTP. ${ }^{14}$ Her income level $y$ is irrelevant. By considering the relative change $\rho=\left(\mathrm{WTA}^{\prime}-\mathrm{WTP}\right) / \mathrm{WTP}$ for a significant set of subjects, one can then use standard statistical methods to test the hypothesis that $\rho=0$ (or that $\rho \geq 0$ ).

REMARK 22: We emphasize that in order to test the normative equivalence between $C$ and $E$, it is not possible to simply average subject responses over half-cycles as it is common practice in the extant literature. A full-cycle response (e.g., WTP' and WTA) needs to be elicited from each individual. Then the empirical hypothesis that the two are equal can be tested based on a representative sample of individuals.

REMARK 23: In order to compare WTP and WTA' from an agent, one can use an augmented version of the well-known Becker-DeGroot-Marschak (BDM) mechanism (Becker, DeGroot, and Marschak 1964) by Weber (2009). The augmented BDM mechanism takes into account the impossibility of extracting all rent from an agent with private information to decrease income by WTP before eliciting WTA'. It uses the standard BDM mechanism to elicit the first welfare measure, and then, as a function of the realized BDM transfer (yet unknown to the subject), the other welfare measure. ${ }^{15}$ From this, one can obtain empirically testable relations for the endowment effect as a behavioral anomaly.

\section{Discussion}

The measures $C$ (or WTP) and $E$ (or WTA) are central for the evaluation of welfare changes. Which welfare measure to apply depends on the viewpoint of the observer, be it before or after the welfare change takes place. Since this choice of viewpoint may be subjective, it is important to understand systematic differences between $C$ and $E$, which for given income levels are referred to as normative (anti-) endowment effects. Endowment effects characterize the difference between $C$ and $E$, and thus the effect of a change of the evaluation perspective from ex post to ex ante or vice versa. When viewed as a function of income, $C$ and $E$ can be derived from each other, and either of them can be equivalently replaced by a compensatedincome function (cf. Remark 3).

From a normative viewpoint, the intimate relationship between the monotonicity of the welfare measures and the presence of the normative endowment effect is remarkable. Similar to Quah's (2007) conditions of supermodularity and (partial) concavity of the agent's utility function as sufficient for normal consumption in

\footnotetext{
${ }^{14}$ Alternately, one can traverse the transaction cycle in the opposite direction by first endowing the subject with the nonmarket good, e.g., by offering a complimentary coffee mug, then eliciting the subject's WTA for giving up the mug, implementing the transaction, and finally eliciting the subject's WTP' (at the compensated income level) for returning to her endowed state. Then, again, from a normative point of view $\mathrm{WTP}^{\prime}=$ WTA must be satisfied.

15 The standard BDM mechanism achieves truthful revelation of a private value by asking the subject to announce her WTP (or WTA) for an object, given that the actual payment amount is the realization of a random variable (with sufficiently large support) and that a transaction takes place only if ex post the realized transfer $t$ is compatible with the subject's announcement (i.e., if $t \leq$ WTP or $t \geq$ WTA).
} 
a continuous context, we obtain that essentially the same conditions yield monotonicity of the welfare measures, which in turn implies the normative endowment effect (i.e., that $C \leq E$ ) with respect to a discrete state change. Examples 12 and 15 showed the relevance of these insights in simple market settings. Indeed, if WTP is nondecreasing in income the endowment effect obtains, explaining, among other things, the possibility of no trade at intermediate income levels. Endowment effects also obtain in the neighborhood of indifference points, which in Example 20 was related to the presence of income effects in a simple information acquisition problem involving an investor with constant absolute risk aversion. Lastly, we have shown that if the difference between the two welfare measures is independent of income, then $C=E$ and the agent's underlying indirect utility function must be additively separable.

In our discussion, we separate empirical observations from normative results by distinguishing the normative endowment effect (or anti-endowment effect) from the behavioral endowment effect that has been observed empirically. Before being able to designate the presence (or absence) of a behavioral endowment effect as an anomaly contradicting neoclassical choice theory, it is necessary to characterize normative endowment effects and then test the resulting characterization conditions empirically. A key result of our analysis is that observations contradicting $C(y) \equiv E(y-C(y))$ or $E(y) \equiv C(y+E(y))$ should be considered a behavioral anomaly. Empirical observations suggesting that these relations are violated could not be explained by the normative endowment effect, for it would imply the irreversibility of the compensated-income function in the sense that $w_{10}\left(w_{01}(y)\right) \neq$ $w_{01}\left(w_{10}(y)\right)$. At present, it appears that experimental data for full transaction cycles is needed to specifically test the reversibility of the compensated-income function.

\section{Appendix: Proofs}

\section{PROOF OF PROPOSITION 18:}

We show that the normative endowment effect is present to the right of the indifference point $y_{0}$. Clearly, $v_{0}\left(y_{0}\right)=v_{1}\left(y_{0}\right)$ implies that $C\left(y_{0}\right)=E\left(y_{0}\right)=0$. As a consequence of the desirability of the change from $q_{0}$ to $q_{1}$, the welfare measures $C(y)$ and $E(y)$ are nonnegative for any income level $y$. There are two cases: ${ }^{16}$ either there exists $\varepsilon>0$, such that $C(y)=0$ for all $y \in\left(y_{0}, y_{0}+\varepsilon\right)$, or there is $\varepsilon>0$, such that $C(y)>0$ for all $y \in\left(y_{0}, y_{0}+\varepsilon\right)$. In the first case, the claim of the proposition follows immediately by setting $y_{1}=y_{0}+\varepsilon$. Consider therefore the more interesting second case, and let $\delta_{0} \in\left(y_{0}, y_{0}+\varepsilon\right)$. We then consider a sequence $\delta=\left\{\delta_{k}\right\}_{k=1}^{\infty}$, such that $\delta_{k}=v_{1}^{-1}\left(v_{0}\left(\delta_{k-1}\right)\right)$ for all $k \geq 1$. Since $v_{1}(y)>v_{0}(y)$ on $\left(y_{0}, y_{0}+\varepsilon\right)$, we have that $\delta$ is a decreasing sequence, i.e., $\delta_{k}<\delta_{k-1}$ for all $k \geq 0$. As the sequence $\delta$ is also bounded from below, it converges. The only possible limit is $\delta_{\infty}=0$, as otherwise we obtain by the continuity of $v_{0}$ and $v_{1}$ the contradictory statement that $0<v_{1}^{-1}\left(v_{0}\left(\delta_{\infty}\right)\right)<\delta_{\infty}=\lim _{k \rightarrow \infty} v_{1}^{-1}\left(v_{0}\left(\delta_{k}\right)\right)=v_{1}^{-1}\left(v_{0}\left(\delta_{\infty}\right)\right)$.

\footnotetext{
${ }^{16}$ Because of the assumption that $v_{0}, v_{1}$ are analytic, a third case, that in any right-neighborhood of $y_{0}$ there are countably many roots of $v_{1}(y)-v_{0}(y)$, cannot arise (cf. footnote 10).
} 
We are now ready to conclude by a contradiction argument. Assume that there is an income level $\hat{y}>y_{0}$ with $\hat{y}-y_{0}<\varepsilon$, such that $C(y)>E(y)$ for all $y \in\left(y_{0}, \hat{y}\right)$, i.e., such that the normative endowment effect is not present in a right-neighborhood of the origin. Then, taking the subsequence $\left\{\delta_{k}\right\}_{k \geq n}^{\infty}$ of $\delta$, such that $\delta_{n}<\hat{y}$ for all $k \geq n$, we obtain for any $k \geq n+1$ that

$$
E\left(\delta_{k}\right)=\delta_{k-1}-\delta_{k}<\delta_{k}-\delta_{k+1}=C\left(\delta_{k}\right)
$$

which contradicts the earlier established fact that the sequence $\delta$ is strictly decreasing. Hence, for incomes in a right-neighborhood of the origin the normative endowment must hold. The proof that a normative anti-endowment effect is present for incomes in a left-neighborhood of the indifference point $y_{0}$ is symmetric and therefore omitted.

\section{PROOF OF PROPOSITION 21:}

The proof proceeds in four steps.

1. (i) $\Rightarrow$ (iii): Assume that $E(y) \equiv C(y)+\kappa$ for some given real constant $\kappa$. By Proposition 2 and equation (3a), it is

$$
C(y)=E\left(w_{01}(y)\right)=C\left(w_{01}(y)\right)+\kappa=C(y-C(y))+\kappa
$$

for any income $y$. Thus, we have to prove that

$$
C(y) \equiv C(y-C(y))+\kappa \Rightarrow C(y) \equiv \text { const. }
$$

The following lemma establishes that (11) is satisfied if $C$ is a polynomial of degree $N \geq 1$.

LEMMA 24: If $N \geq 1$ and $c_{N}(y)=\sum_{n=0}^{N} a_{n} y^{n}\left(\right.$ for some $\left.a_{0}, a_{1}, \ldots, a_{N} \in \mathbb{R}\right)$, then:

$$
c_{N}(y) \equiv c_{N}\left(y-c_{N}(y)\right)+\kappa \Rightarrow c_{N}(y) \equiv a_{0}
$$

PROOF OF LEMMA 24 (BY INDUCTION):

For $N=1$, it is $c_{1}(y)-c_{1}\left(y-c_{1}(y)\right) \equiv a_{1}\left(a_{0}+a_{1} y\right)+\kappa$, so that (12) holds for $N=1$. Assume now that (12) holds for some $N \geq 1$. Then,

$$
\begin{aligned}
c_{N+1}(y)-c_{N+1}\left(y-c_{N+1}(y)\right)= & c_{N}(y)+a_{N+1} y^{N+1} \\
& -c_{N}\left(y-c_{N}(y)-a_{N+1} y^{N+1}\right) \\
& -a_{N+1}\left(y-c_{N}(y)-a_{N+1} y^{N+1}\right)^{N+1} \\
& (N+1)^{2}-1 \\
= & \sum_{n=0} b_{n} y^{n}-a_{N+1}\left(-a_{N+1}\right)^{N+1} y^{(N+1)^{2}}
\end{aligned}
$$


for some real coefficients $b_{0}, b_{1}, \ldots, b_{(N+1)^{2}-1}$, so that $c_{N+1}(y)-c_{N+1}\left(y-c_{N+1}(y)\right)$ $\equiv \kappa$ implies that $a_{N+1}=0$. This in turn implies that

$$
c_{N+1}(y)-c_{N+1}\left(y-c_{N+1}(y)\right)=c_{N}(y)-c_{N}\left(y-c_{N}(y)\right),
$$

so that by (12) $a_{1}=a_{2}=\cdots=a_{N}=0$. Hence, the implication (12) holds for $N+1$ if it holds for $N$, which completes the proof of Lemma 24.

Lemma 24 directly implies that (11) must hold for any entire function $C(y)$, as it can be globally represented by a Taylor power series. ${ }^{17}$ Lastly, it is well known that the class of entire functions is dense in the class of continuous functions, i.e., any continuous function $C(y)$ can be approximated, on a given compact set $[y, \bar{y}]$ and for a given $\varepsilon>0$, by an entire function $\hat{C}_{\varepsilon}(y)$, such that ${ }^{18}$

$$
\sup _{y \in[\underline{y}, \bar{y}]}\left|C(y)-\hat{C}_{\varepsilon}(y)\right| \leq \varepsilon .
$$

Naturally, the function $\hat{C}_{\varepsilon}(y)$ does not generally satisfy the relation (10), but it can be selected such that it is approximately satisfied on an interval $\mathcal{Y}=[\underline{y}, \bar{y}]$, such that ${ }^{19}$

$$
\left|\hat{C}_{\varepsilon}(y)-\hat{C}_{\varepsilon}(0)\right| \leq \varepsilon / 3
$$

for all $y \in \mathcal{Y}$. Assume now that there exist income levels $y_{1}, y_{2}$ such that $C\left(y_{1}\right) \neq C\left(y_{2}\right)$. Without any loss of generality, we can set $y_{2}=0$ (the general case then follows by a simple translation). If we set $\varepsilon=\left|C\left(y_{1}\right)-C(0)\right| / 4>0$, we obtain an immediate contradiction, because $\hat{C}_{\varepsilon}$ cannot at the same time satisfy inequalities (13) and (14), where $y=\min \left\{0, y_{1}\right\}$ and $\bar{y}=\max \left\{0, y_{1}\right\}$. Hence, $C$ must be independent of income, and by Proposition 2 the equivalent variation $E$ is also independent of income.

2. (iii) $\Rightarrow$ (ii): If both $C$ and $E$ are independent of income, then Proposition 2(i) implies immediately that $C(y) \equiv E(y-C(y)) \equiv E(y)$.

3. (ii) $\Rightarrow$ (iv): Assume that $C(y) \equiv E(y)$. Then, by Step 1, the welfare measures $C$ and $E$ are independent of the agent's income. By choosing a money metric such that $v_{1}(y) \equiv y$ we obtain that $v_{0}(y)=y-C=v_{1}(y)-C$, for any income level $y$. Thus, $v_{1}(y)-v_{0}(y) \equiv$ const.

\footnotetext{
${ }^{17}$ It is clear that (11) holds for any truncation of the series, and thus must also hold in the limit, since the power series converges (to $C(y)$ ).

${ }^{18}$ The Weierstrass approximation theorem (see, e.g., Serge Lang 1993, 229) guarantees this even for polynomials, a subclass of entire functions.

${ }^{19}$ The corresponding precise arguments are tedious but standard and therefore omitted.
} 
4. (iv) $\Rightarrow(\mathrm{i})$ : Let $v_{0}(y) \equiv y$ and $v_{1}(y)-v_{0}(y) \equiv \lambda$ for some given (nonnegative) constant $\lambda$. By equations (1a) and (1b), it is therefore

$$
y=v_{1}(y-C(y))=\lambda+y-C(y)
$$

and

$$
v_{1}(y)=\lambda+y=y+E(y)
$$

for all income levels $y$, so that $C(y) \equiv E(y) \equiv \lambda$. The argument proceeds analogously if a money metric is chosen such that $v_{1}(y) \equiv y$ and $v_{0}(y)$ $\equiv v_{1}(y)-\lambda \equiv y-\lambda$.

Steps $1-4$ establish that $(\mathrm{i}) \Rightarrow($ iii $) \Rightarrow$ (ii) $\Rightarrow$ (iv) $\Rightarrow($ i), which concludes our proof.

\section{REFERENCES}

Afriat, S. N. 1967. “The Construction of Utility Functions from Expenditure Data.” International Economic Review, 8(1): 67-77.

Becker, Gordon M., Morris H. DeGroot, and Jacob Marschak. 1964. "Measuring Utility by a SingleResponse Sequential Method." Behavioral Science, 9(3): 226-32.

Blackwell, David H. 1951. "Comparison of Experiments.” In Proceedings of the Second Berkeley Symposium on Mathematical Statistics and Probability, ed. Jerzy Neyman, 93-102. Berkeley, CA: University of California Press.

Chambers, Christopher P., and Federico Echenique. 2009. "Supermodularity and Preferences." Journal of Economic Theory, 144(3): 1004-14.

-Chipman, John S. 1977. "An Empirical Implication of Auspitz-Lieben-Edgeworth-Pareto Complementarity." Journal of Economic Theory, 14(1): 228-31.

Edgeworth, Francis Y. 1897. "La Teoria Pura del Monopolio.” Giornale degli Economisti, 2nd Ser., (15): 13-31, 307-20, 405-14.

Hammack, Judd, and Gardner Mallard Brown, Jr. 1974. Waterfowl and Wetlands: Toward Bioeconomic Analysis. Baltimore, MD: Johns Hopkins University Press.

Hanemann, W. Michael. 1991. "Willingness to Pay and Willingness to Accept: How Much Can They Differ?" American Economic Review, 81(3): 635-47.

Hicks, J. R., and R. G. D. Allen. 1934. "A Reconsideration of the Theory of Value. Part I." Economica, 1(1): 52-76.

Hicks, John R. 1939. Value and Capital. Oxford, UK: Clarendon Press.

Kahneman, Daniel, Jack L. Knetsch, and Richard H. Thaler. 1991. "Anomalies: The Endowment Effect, Loss Aversion, and Status Quo Bias.” Journal of Economic Perspectives, 5(1): 193-206.

Kahneman, Daniel, and Amos Tversky. 1979. "Prospect Theory: An Analysis of Decision under Risk." Econometrica, 47(2): 263-91.

Kihlstrom, Richard E. 1984. "A Bayesian Exposition of Blackwell's Theorem on the Comparison of Experiments." In Bayesian Models in Economic Theory, ed. Marcel Boyer and Richard E. Kihlstrom, 13-31. Amsterdam: North-Holland.

Knetsch, Jack L. 1989. "The Endowment Effect and Evidence of Nonreversible Indifference Curves." American Economic Review, 79(5): 1277-84.

Knetsch, Jack L., and J. A. Sinden. 1984. "Willingness to Pay and Compensation Demanded: Experimental Evidence of an Unexpected Disparity in Measures of Value." Quarterly Journal of Economics, 99(3): 507-21.

Lang, Serge. 1993. Real and Functional Analysis. 3rd ed. Graduate Texts in Mathematics. New York, NY: Springer.

Milgrom, Paul, and Chris Shannon. 1994. "Monotone Comparative Statics." Econometrica, 62(1): 157-80.

Pareto, Vilfredo. 1909. Manuel d'Économie Politique. Giard and Brière, Paris, France. 
Plott, Charles R., and Kathryn Zeiler. 2005. “The Willingness to Pay-Willingness to Accept Gap, The "Endowment Effect," Subject Misconceptions, and Experimental Procedures for Eliciting Valuations." American Economic Review, 95(3): 530-45.

Plott, Charles R., and Kathryn Zeiler. 2007. "Exchange Asymmetries Incorrectly Interpreted as Evidence of Endowment Effect Theory and Prospect Theory?" American Economic Review, 97(4): 1449-66.

Quah, John K.-H. 2007. “The Comparative Statics of Constrained Optimization Problems.” Econometrica, 75(2): 401-31.

Randall, Alan, and John R. Stoll. 1980. “Consumer's Surplus in Commodity Space.” American Economic Review, 70(3): 449-55.

Samuelson, Paul A. 1947. Foundations of Economic Analysis. Cambridge, MA: Harvard University Press.

Schulze, William D., Ralph C. d'Arge, and David S. Brookshire. 1981. "Valuing Environmental Commodities: Some Recent Experiments." Land Economics, 57(2): 151-72.

Thaler, Richard H. 1980. "Toward a Positive Theory of Consumer Choice." Journal of Economic Behavior and Organization, 1(1): 36-60.

Topkis, Donald M. 1998. Supermodularity and Complementarity. Princeton, NJ: Princeton University Press.

-Weber, Thomas A. 2003. "An Exact Relation between Willingness to Pay and Willingness to Accept." Economics Letters, 80(3): 311-15.

Weber, Thomas A. 2009. "An Augmented Becker-DeGroot-Marschak Mechanism for Transaction Cycles." Unpublished.

Willig, Robert D. 1976. "Consumer's Surplus without Apology." American Economic Review, 66(4): 589-97. 
This article has been cited by:

1. Andrea Mantovi. 2013. On the commutativity of expansion and substitution effects. Journal of Economics . [CrossRef]

2. Thomas A. Weber. 2011. An augmented Becker-DeGroot-Marschak mechanism for transaction cycles. Economics Letters . [CrossRef] 